\title{
Absent Bell's phenomenon in patients with thyroid eye disease
}

\author{
Hyun Woo Chung ${ }^{1,2}$, Hwa Lee ${ }^{1 *}$ (D) and Sehyun Baek ${ }^{3}$
}

\begin{abstract}
Background: To investigate the incidence of absent Bell's phenomenon (BP) and the relationship between absent $\mathrm{BP}$ and inferior rectus muscle hypertrophy and other clinical features in patients with thyroid eye disease (TED).

Methods: A total of 104 patients who were first diagnosed with TED between January and December 2014 were included. Inferior rectus muscle area and associations with clinical features of TED and thyroid function test including thyroid specific antibodies were compared between patients with TED with and without BP. The volume of the inferior rectus muscle was calculated by adding up all the cross-sectional areas measured on sagittal $\mathrm{CT}$ images.

Results: Among the 104 patients, 14 had absent BP (13.5\%), 12 with bilateral and two with unilateral. There was no significant difference in thyroid function test, presence of TSIs, exophthalmos, or volume of inferior rectus muscle measured in $C T$ scans $(P>0.05)$. Incidence of diplopia, elevation limitation, and upper eyelid retraction were risk factors of absent BP in TED patients (by logistic regression analysis, $P<0.05$ ).

Conclusions: Inferior rectus muscle hypertrophy was not the cause of absent BP in TED patients. Fibrosis and tightening of the inferior rectus muscle, lower eyelid, and surrounding orbital tissues, rather than inferior rectus muscle hypertrophy, might be related to absent BP in TED patients.
\end{abstract}

Keywords: Bell's phenomeon, Fibrosis, Inferior rectus muscle, Thryoid eye disease

\section{Background}

The clinical features of thyroid eye disease (TED) consist of a variable combination of eyelid retraction, eyelid swelling, proptosis, impaired ocular motility, keratitis, exposure keratopathy, and optic nerve compression $[1,2]$. One of the leading causes of ocular surface damage in TED is dry eye syndrome [3]. Factors considered to cause dry eye in TED include exophthalmos, increasing palpebral fissure height, lagophthalmos, and reduced tear production $[4,5]$.

Sight-threatening corneal ulceration is far less common than dysthyroid optic neuropathy and presents as corneal staining, sometimes with thinning and very occasionally with corneal perforation [6]. Recently reported

\footnotetext{
* Correspondence: lifa98@korea.ac.kr

'Department of Ophthalmology, Ansan Hospital, Korea University College of Medicine, 123 Jeokkumro, Ansan-shi, Kyunggi-do 15355, Republic of Korea Full list of author information is available at the end of the article
}

incidence of corneal ulcer in thyroid eye disease patients was $1.3 \%$ [7]. In addition, corneal exposure and ulceration could take place when the eyelids are incompletely closed due to lagophthalmos and there is no Bell's phenomenon (BP). Hence, absent BP with lagophthalmos increases the risk of corneal damage.

Absence of BP was first reported by Charles Bell in 1823 as an upward deviation of the eye during forcible eyelid closure with a lower motor neuron defect of the facial nerve [8]. Although the physiological mechanism has not been fully explained, normal function of extraocular muscles is essential for this sign. Although BP is absent in $10 \%$ individuals, it is more likely to be lost in TED patient with very tight inferior rectus muscle limiting upward movement of the eyeball [9]. However, the exact incidence of loss of this reflex and whether BP is correlated with inferior right rectus muscle hypertrophy, 
tight rectus muscle, or other clinical factors have not been studied.

The purpose of this study is to investigate the incidence of absent BP and the relationship between absent $\mathrm{BP}$ and inferior rectus muscle hypertrophy and other clinical features in patients with TED.

\section{Methods}

After receiving approval from the Institutional Review Board at Korea University College of Medicine Guro and Ansan Hospital, 104 patients who were diagnosed with TED in accordance with the diagnostic criteria of the American Academy of Ophthalmology, from January to December 2014, were enrolled in this cross-sectional study [10]. The study was conducted in accordance with the Declaration of Helsinki. Patients who had undergone eyelid surgery, radiation therapy, decompression, or strabismus surgery were excluded. Patients who had an incomplete set of CT (computed tomography) images were also excluded.

Laboratory measurements for TSH (Thyroid Stimulating Hormone), FT4(Free T4), T3, and thyroid specific antibodies, including thyroid peroxidase antibodies (TPOAbs) and thyroid-stimulating immunoglobulin (TSI), were assessed.

The following clinical features were investigated: age, sex, thyroid status and systemic treatment, bestcorrected visual acuity (BCVA), color vision, lid swelling, upper and lower eyelid retraction, lid lag, keratopathy, lagophthalmos, exophthalmos with a Hertel exophthalmometer (Oculus; Oculus Optik Geraet, Wetzlar, Germany), diplopia score according to the Gorman, [11] abduction, adduction, elevation, depression, Clinical Activity Score (CAS), [12] and modified NOSPECS classification [13]. Upper eyelid retraction was defined as the upper eyelid at or above the superior limbus in primary position without frontalis muscle contraction, while lower eyelid retraction was position of the lower eyelid below the inferior limbus in primary position [14].
Symptoms of diplopia were recorded based on BahnGorman progression: 0 as no diplopia, 1 as intermittent diplopia (present with fatigue), 2 as inconstant diplopia (with vertical or horizontal gaze), 3 as constant diplopia in straight gaze that is correctable with prisms, and 4 as constant diplopia that is not correctable with prisms [15]. The degree of elevation limitation as a sign of inferior rectus involvement was graded on a scale of -1 to $4 ;-4$ indicates no elevation movement, -3 indicates $25 \%$ movement remains, -2 indicates $50 \%$ movement remains, and -1 indicates $75 \%$ movement remains. CAS consisted of the following seven items: retrobulbar pain with movement and/or rest, redness of the eyelids and/ or conjunctivae, swelling of the caruncle, swelling of the eyelids, or chemosis. A CAS score greater than 4 was considered active disease [9].

BP was assessed by gently lifting each upper lid manually while the patients attempted gentle closure while observing closely whether upward rotation takes place. If there was no upward rotation, then it was regarded absent BP (Fig. 1A). Non-contrast orbital CT was performed on a 128 row multi-detector CT (Ingenuity Core 128, Philips Healthcare, Cleveland, USA) with $3 \mathrm{~mm}$ thickness of axial, coronal, and sagittal images. The cross-sectional areas of the inferior rectus muscle (IR) were measured by tracing outlines of each tissue on sagittal CT images using a PACS system (PiViewSTAR, INFINITT, Korea) (Fig. 1B). The volume of the IR was calculated by adding up all the cross-sectional volumes in the same manner as previous studies [16, 17].

Presence of BP was investigated in control group who visited the clinic and diagnosed with dry eye syndrome, age and sex matched as TED patients.

For statistical analyses, SPSS software version 18.0 (SPSS Inc., Chicago, IL) was used. To analyze differences in the independent variables, Fisher's exact test was used. To analyze differences in continuous variables, Mann-Whitney U-test was used in the case of an
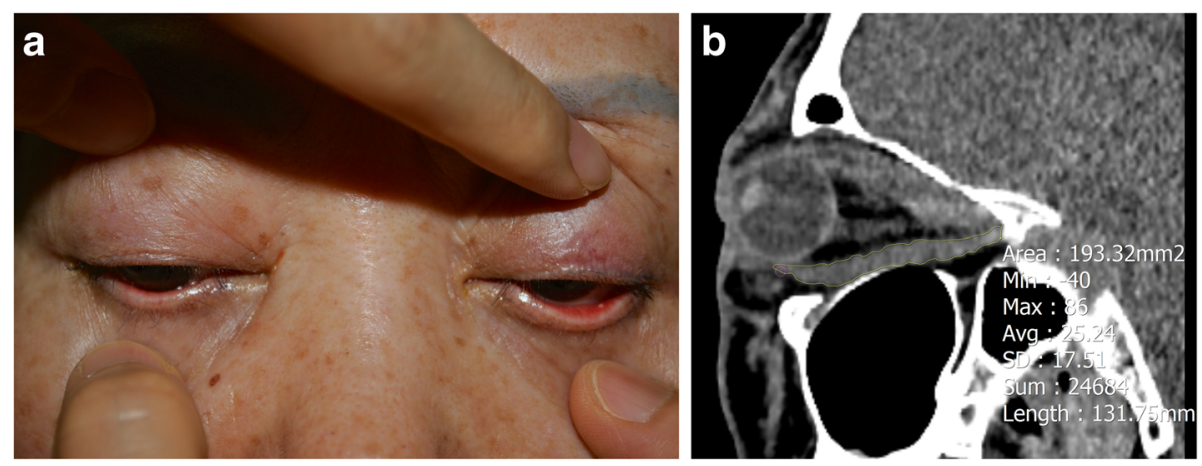

Fig. 1 A Bell's phenomenon is assessed by gently lifting each upper lid manually while the patient's eyes are closed. The examination shows absent BP in both eyes. B Measurement of cross-sectional areas of inferior rectus muscle on sagittal CT images 
abnormal distribution. We used multivariate logistic regression analysis to assess absent BP risk factors including presence of diplopia, elevation limitation, and the incidence of upper eyelid retraction were included. $P$-values less than 0.05 were considered statistically significant.

\section{Results}

During the enrollment period, a total of 104 patients were diagnosed with TED at Korea University Guro Hospital and Korea University Ansan Hospital. Among them, 14 patients showed negative BP (13.5\%), 12 were bilateral, and two were unilateral. Patient demographics and thyroid dysfunctions are shown in Table 1. There was a female predominance with either absent or positive BP. The mean age was higher in absent BP patients $(54.0 \pm 11.9$ years $)$ than in positive BP patients $(44.1 \pm$ 14.9 years), but the difference was not significant $(P=$ 0.15 , by Mann-Whitney $U$ test). Thyroid dysfunction was noted in $28 \%$ and positive TSI was noted in $34 \%$ of patients with absent BP. (Table 1) The differences in thyroid dysfunction, TPOAbs, TSI, thyroid treatment including antithyroid medication and radioactive iodine between patients with positive BP and patients with negative $\mathrm{BP}$ were not statistically significant $(P>0.05)$.

The mean exophthalmometry values were $14.7 / 15.8 \mathrm{~mm}$ in absent BP patients (right/left, range 10.0-28.0) and 16.9/ $16.6 \mathrm{~mm}$ in positive BP patients (range 13.0-21.0) $(p=$ $0.179,0.504$ ) (Table 2). Clinically significant active disease, defined as a CAS $>4 / 10$, was not present in negative BP patients. The CAS score was the same at 1.2 in BP absent and positive patients. The mean NOSPECS score was 2.0 in absent BP patients and 2.6 in positive BP patients.

Presence of diplopia was $50 \%$ in absent BP patients and $18 \%$ in positive BP patients $(P=0.007$ by logistic regression analysis, Odds ratio $7.395 \% \mathrm{CI})$. Elevation limitation was $43 \% / 43 \%$ (right/left) in absent BP patients and $16 \% / 18 \%$ in positive BP patients (right/left) $(P=$ $0.016 / 0.033$, Odds ratio $5.8 / 4.695 \% \mathrm{CI})$. The incidence of upper eyelid retraction was $64 \% / 64 \%$ (right/left) in absent $\mathrm{BP}$ and $13 \% / 16 \%$ in positive $\mathrm{BP}$ patients $(P=0.000 /$ 0.000 , Odds ratio $21.1 / 16.795 \% \mathrm{CI}$ ).

Mean volume of inferior rectus muscle was $1148.7 \pm$ $198.6 / 1069.9 \pm 181.1 \mathrm{~mm}^{3}$ (right/left) in positive BP patients and $1458.4 \pm 162.9 / 1389.5 \pm 179.3 \mathrm{~mm}^{3}$ in absent BP patients $(P=0.311 / 0.190)$.

In control group, dry eye syndrome patients with age and sex matched as TED patients, incidence rate of absent BP was $11.5 \%$ (12 patients out of 104 patients). In absent BP group, 7 patients were male (58.3\%). The mean age was higher in BP absent patients $(51.3 \pm 10.4$ years) than in BP positive patients ( $44.6 \pm 15.3$ years). The difference of the absent $\mathrm{BP}$ incidence was not statistically significant $(P=0.675)$.

\section{Discussion}

Charles Bell encountered many cases of unilateral paralysis of the facial muscles and noted that the eyeball on the paralyzed side invariably rotates upward when the patient tries to close her/his eyelids [18]. This palpebraloculogyric reflex, which is more noticeable when eyelid closure is incomplete, is known as BP. Although this reflex is absent in $10 \%$ of individuals, it is more likely to be absent in TED patient who has a very tight inferior rectus [9]. However the incidence of absent BP in TED patients was $13.5 \%$ and not significantly higher to that of the control group in our study (11.5\%).

TED is an immune-mediated inflammatory disorder that causes expansion of the extraocular muscles and orbital fat from edema with deposition of collagen and glycosaminoglycans $[19,20]$. The typical course of a TED patient without specific treatment is initial active

Table 1 Characteristics of thyroid eye disease patients according to presence of Bell's phenomenon

\begin{tabular}{llll}
\hline & Bell's phenomenon + & Bell's phenomenon - & p-value \\
\hline Patients [N (\%)] & $90(86.5)$ & $14(13.5)$ & $54.0 \pm 11.9(19 \sim 66)$ \\
Age (years) & $44.1 \pm 14.9(20 \sim 66)$ & & 0.15 \\
Sex [N (\%)] & & $2(14)$ & $12(86)$ \\
$\quad$ Male & $28(31)$ & $23 \pm 9.9$ & 0.196 \\
$\quad$ Female & $62(69)$ & $4(29 \%)$ & 0.23 \\
Disease duration (months) & $12 \pm 14.4$ & $4(29 \%)$ & 0.645 \\
Thyroid dysfunction & $25(28 \%)$ & $2(14 \%)$ & 0.607 \\
Antithyroid medications & $24(27 \%)$ & $2(14 \%)$ & 0.225 \\
RAl & $4(4 \%)$ & $5(36 \%)$ & 0.828 \\
TPO Abs & $11(12 \%)$ & 0.926 \\
TSI & $31(34 \%)$ &
\end{tabular}

By Fisher's exact test and Mann-Whitney U-test

Abbreviations: RAI Radioactive iodine, TPO Abs Thyroid peroxidase antibodies, TSI Thyroid-stimulating immunoglobulin 
Table 2 Analysis of risk factors in clinical characteristics for absent Bell's phenomenon

\begin{tabular}{llll}
\hline & Bell's phenomenon + & Bell's phenomeon - & $p$-value \\
\hline Exophthalmometry (Rt/Lt) & $16.9 / 16.6$ & $14.7 / 15.8$ & $0.179 / 0.504$ \\
CAS score (Rt/Lt) & $1.2 \pm 1.2$ & $1.2 \pm 0.8$ & 0.895 \\
Active phase & $10(11.1 \%)$ & $0(0 \%)$ & 0.190 \\
NOSPECS score & $2.6 \pm 2.3$ & $2.0 \pm 1.6$ & 0.554 \\
Lagophthalmos & $14(16 \%)$ & $4(40 \%)$ & 0.231 \\
Presence of diplopia & $16(18 \%)$ & $7(50 \%)$ & $0.007^{*}$ \\
Bahn-Gorman score & 0.4 & 1.4 & 0.341 \\
Elevation limitation (Rt/Lt) & $14 / 16(16 \% / 18 \%)$ & $6 / 6(43 \% / 43 \%)$ & $0.016 / 0.033^{*}$ \\
Eyelid retraction (Rt/Lt) & Upper 12/14 (13\%/16\%) & $0.000 / 0.000^{*}$ \\
Volume of inferior rectus muscle $\left(\mathrm{mm}^{3}\right)(\mathrm{Rt} / \mathrm{Lt})$ & Lower 10/16 (11\%/18\%) & $0 / 3(0 \% / 21 \%)$ & $0.190 / 0.742$ \\
\hline
\end{tabular}

by Multivariate logistic regression analysis, $p$-value $<0.05^{*}$

progressive phase, followed by a phase of spontaneous slow improvement described by Rundle [21].

After regression of the inflammatory process, fibrosis may develop; affected tissues may display traits such as proptosis; and eyelid retraction and chronic dysfunction of extraocular muscles might persist and not return to their previous normal functioning state.

In our study, there were none of the patients with absent BP had active TED. Although mean volume of inferior rectus muscle in absent BP group was larger than that of positive BP group, the difference was not statistically significant. However, incidence of diplopia, elevation limitation, and upper eyelid retraction in TED patients without BP were significantly different from those with BP. These suggest that absent BP is more likely to be related to fibrosis and tightening of the orbital tissues rather than active inflammation and orbital tissue and rectus muscle hypertrophy.

TED patients can be divided into four groups: no fat volume or muscle volume increase, only fat volume increase, only muscle volume increase, and both fat and muscle volume increases [22]. Increase in muscle volume was present in the largest group and was related to older age, more proptosis, and reduced duction values [23]. Although extraocular muscle volume could have a relationship with duction limitation and more proptosis, fibrosis and tightening of the extraocular muscle and eyelid retractors rather than volume increase itself could exert more influence on extraocular movement and the normal protective BP.

Eyelid retraction can involve the upper or lower eyelid and has been suggested as the most common sign and one of the prime diagnostic criteria for TED [15]. The cause of upper eyelid retraction is multifactorial as follows; increased sympathetic tone in Müller's muscle, levator muscle fiber enlargement, levator muscle contracture or fibrosis, relative or absolute exophthalmos, or scarring and/or inflammation of the septum and anterior lamella [24, 25]. In addition, tight restriction of the inferior rectus muscle leads to upper eyelid retraction, regardless of upper eyelid pathology [15]. It causes resultant increase in tone of the superior rectus and levator muscle [26].

In our study, the absent BP patients were more likely have elevation limitation and upper eyelid retraction and this could be explained by the fixation duress. Fixation duress was presumed to play an additional role in the pathogenesis of upper eyelid retraction in patients with profound restriction of elevation from the primary position and inferior rectus muscle restriction [26-28]. Upper eyelid retraction clinically apparent when fixating in the primary position or attempting upgaze resolved on downgaze. Longstanding contraction of the levator muscle due to this fixation duress may result in shortening and contracture of the levator muscle that does not readily resolve on relief of the inferior rectus muscle restriction [29].

Limitation of this study is the presence of weak but positive BP. Patients were classified roughly into 2 groups, namely presence or absence of BP. The group designated as positive $\mathrm{BP}$ is suspected to have included many patients with weak but positive BP, probably resulting into no statistically significant difference in some factors between the groups. To measure the amount of BP and analyze the influential factors may provide more information. Another limitation is the measurement method of the IR volume. There has been various measurement method including calculating cross-sectional area in coronal Magnetic Resonance (MR) imaging using maximum diameters approximately $1 \mathrm{~cm}$ behind posterior pole of globe [30] and crosssectional areas of the IR by tracing outlines of each tissue on sagittal CT and MR images [16, 17]. The latter method was used in this study. Although no consensus 
exists regarding the quantification of EOM size in TED but various volumetric analysis might provide another information.

The combination of eyelid retraction and proptosis in TED increases corneal exposure and may lead to symptoms of irritation, photophobia, secondary epiphora, and blurred vision [31]. Corneal ulceration arises from lagophthalmos and corneal exposure due to proptosis, lower eyelid retraction, and/or poor levator function, usually accompanied by a tight inferior rectus [32]. Although the incidence of absent BP in TED patients is not higher than that of control group in our study, it has to be investigated further if corneal exposure or ulceration occur more often in patients having both absent $\mathrm{BP}$ and lagophthalmos vs. only absent BP.

The test for the presence of BP is not a routinely examined in clinical settings, so more attention should be paid to it especially in patients with lagophthalmos. We recommend that presence/ absence of BP should be carefully performed in patients with TED especially those with lagophthalmos.

\section{Conclusion}

In conclusion, we reported the prevalence (13.5\%) of absent BP in 104 Korean patients with TED. Inferior rectus muscle hypertrophy was not the cause of absent $\mathrm{BP}$ in TED patients. Instead, fibrosis and tightening of the inferior rectus muscle, eyelid, and surrounding orbital tissues might be related to absent BP in TED patients.

\section{Abbreviations}

BP: Bell's phenomenon; CAS: Clinical Activity Score; FT4: Free T4; IR: Inferior rectus muscle; TED: Thyroid eye disease; TPOAbs: Thyroid peroxidase antibodies; TSH: Thyroid stimulating hormone; TSI: Thyroid-stimulating immunoglobulin

\section{Acknowledgments}

Not applicable.

\section{Authors' contributions}

$\mathrm{HL}$ designed the study. $\mathrm{HC}$ analyzed the study and drafted all versions of the manuscript. SB advised on the design and analyses, and reviewed successive drafts of the manuscript. All authors have read and approved the manuscript.

\section{Funding}

This study was supported by a grant from National Research Foundation in Korea (NRF-2017R1C1B5017913). The funder had no influence on the design, collection, analysis or interpretation of the data, or in writing the manuscript.

\section{Availability of data and materials}

The datasets used and analyzed during the current study are available from the corresponding author on reasonable request.

\section{Declarations}

\section{Ethics approval and consent to participate}

The institutional review board of the Korea University Ansan hospital approved the research protocol (K2017-3071-002). Requirement for patient consent was waived by the institutional review board because of the retrospective study design and data anonymization.

\section{Consent for publication}

The written informed consent for publication of identifying patient/clinical data and identifiable images was obtained.

\section{Competing interests}

The authors declare that they have no competing interests.

\section{Author details}

${ }^{1}$ Department of Ophthalmology, Ansan Hospital, Korea University College of Medicine, 123 Jeokkumro, Ansan-shi, Kyunggi-do 15355, Republic of Korea. 2Department of Ophthalmology, Guro Hospital, Korea University College of Medicine, 148 Gurodongro, Seoul 08308, Republic of Korea. ${ }^{3}$ Department of Ophthalmology, Korea University College of Medicine, Seoul, South Korea.

Received: 15 September 2020 Accepted: 13 September 2021

Published online: 11 October 2021

\section{References}

1. Kendler DL, Lippa J, Rootman J. The initial clinical characteristics of Graves' orbitopathy vary with age and sex. Arch Ophthalmol. 1993;111(2):197-201. https://doi.org/10.1001/archopht.1993.01090020051022.

2. Wiersinga WM, Bartalena L. Epidemiology and prevention of Graves' ophthalmopathy. Thyroid. 2002;12(10):855-60. https://doi.org/10.1089/1 05072502761016476

3. Eckstein AK, Finkenrath A, Heiligenhaus A, Renzing-Kohler K, Esser J, Kruger

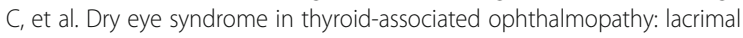
expression of TSH receptor suggests involvement of TSHR-specific autoantibodies. Acta Ophthalmol Scand. 2004;82(3 Pt 1):291-7. https://doi. org/10.1111/j.1395-3907.2004.00268.x.

4. Gilbard JP, Farris RL. Ocular surface drying and tear film osmolarity in thyroid eye disease. Acta Ophthalmol. 1983;61(1):108-16. https://doi.org/1 0.1111/j.1755-3768.1983.tb01401.x.

5. Khurana AK, Sunder S, Ahluwalia BK, Malhotra KC. Tear film profile in Graves' ophthalmopathy. Acta Ophthalmol. 1992;70(3):346-9. https://doi.org/1 0.1111/j.1755-3768.1992.tb08576.x.

6. Barrio-Barrio J, Sabater AL, Bonet-Farriol E, Velazquez-Villoria A, Galofre JC. Graves' Ophthalmopathy: VISA versus EUGOGO classification, assessment, and management. J Ophthalmol. 2015;2015:249125-16. https://doi.org/10.11 $55 / 2015 / 249125$

7. Naik MN, Vasanthapuram VH. Demographic and clinical profile of 1000 patients with thyroid eye disease presenting to a tertiary eye Care Institute in India. Int Ophthalmol. 2021;41(1):231-6. https://doi.org/10.1007/s10792-02 0-01571-6.

8. Bell C. On the motions of the eye, in illustration of the uses of the muscles and nerves of the orbit. Philos Trans R Soc Lond. 1823;113:166-86. https:// doi.org/10.1098/rstl.1823.0017.

9. Wilmar M. Wiersinga GJK: Graves' orbitopathy. 2nd ed. Basel: Karger; 2010. https://doi.org/10.1159/isbn.978-3-8055-9532-2.

10. 2009-2010 Basic and Clinical Science Course, Section 7: Orbit, Eyelids, and Lacrimal System. San Francisco, CA: American Academy of Ophthalmology; 2009.

11. Jacobson DH, Gorman CA. Diagnosis and management of endocrine ophthalmopathy. Med Clin North Am. 1985;69(5):973-88. https://doi.org/1 0.1016/S0025-7125(16)31000-8

12. Mourits MP, Koornneef $L$, Wiersinga WM, Prummel MF, Berghout A, van der Gaag R. Clinical criteria for the assessment of disease activity in Graves' ophthalmopathy: a novel approach. Br J Ophthalmol. 1989;73(8):639-44. https://doi.org/10.1136/bjo.73.8.639.

13. Eckstein AK, Plicht M, Lax H, Neuhauser M, Mann K, Lederbogen S, et al. Thyrotropin receptor autoantibodies are independent risk factors for Graves' ophthalmopathy and help to predict severity and outcome of the disease. J Clin Endocrinol Metab. 2006;91(9):3464-70. https://doi. org/10.1210/jc.2005-2813.

14. Dolman PJ. Evaluating Graves' orbitopathy. Best Pract Res Clin Endocrinol Metab. 2012;26(3):229-48. https://doi.org/10.1016/j.beem.2011.11.007.

15. AM DH A. Ocular Movements. In: DH AAM, Mein J, editors. Diagnosis and Management of Ocular Motility Disorders. 3rd ed. Oxford: Blackwell Science; 2001. p. 104-5.

16. Higashiyama T, Nishida Y, Ohji M. Changes of orbital tissue volumes and proptosis in patients with thyroid extraocular muscle swelling after 
methylprednisolone pulse therapy. Jpn J Ophthalmol. 2015;59(6):430-5. https://doi.org/10.1007/s10384-015-0410-4.

17. Nishida Y, Tian S, Isberg B, Tallstedt L, Lennerstrand G. MRI measurements of orbital tissues in dysthyroid ophthalmopathy. Graefes Arch Clin Exp Ophthalmol. 2001;239(11):824-31. https://doi.org/10.1007/s004170100352.

18. Wilkins RH, Brody IA. Bell's palsy and Bell's phenomenon. Arch Neurol. 1969; 21(6):661-2. https://doi.org/10.1001/archneur.1969.00480180117012.

19. Bahn RS. Graves' ophthalmopathy. N Engl J Med. 2010;362(8):726-38. https://doi.org/10.1056/NEJMra0905750.

20. Garrity JA, Bahn RS. Pathogenesis of graves ophthalmopathy: implications for prediction, prevention, and treatment. Am J Ophthalmol. 2006;142(1): 147-53. https://doi.org/10.1016/j.ajo.2006.02.047.

21. Rundle FF. Management of exophthalmos and related ocular changes in Graves' disease. Metabolism. 1957;6(1):36-48.

22. FW KL Z. Wittebol-post D: quantitative volumetric assessment of orbital soft tissue. Berlin: Springer-Verlag; 1991.

23. Regensburg NI, Wiersinga WM, Berendschot TT, Potgieser P, Mourits MP. Do subtypes of graves' orbitopathy exist? Ophthalmology. 2011;118(1):191-6. https://doi.org/10.1016/j.ophtha.2010.04.004.

24. Grove AS Jr. Upper eyelid retraction and Graves' disease. Ophthalmology. 1981;88(6):499-506. https://doi.org/10.1016/50161-6420(81)34991-4.

25. Small RG. Enlargement of levator palpebrae superioris muscle fibers in Graves' ophthalmopathy. Ophthalmology. 1989;96(4):424-30. https://doi. org/10.1016/50161-6420(89)32874-0.

26. Hamed LM, Lessner AM. Fixation duress in the pathogenesis of upper eyelid retraction in thyroid orbitopathy. A prospective study. Ophthalmology. 1994; 101(9):1608-13. https://doi.org/10.1016/S0161-6420(94)38033-X.

27. Jampolsky A. What can electromyography do for the ophthalmologist? Investig Ophthalmol. 1970;9(8):570-99.

28. Jampolsky A. Symposium on strabismus: trans New Orleans Acad Ophthalmol. St Louis: CV Mosby; 1978.

29. Grove AS Jr. Orbital disease: examination and diagnostic evaluation. Ophthalmology. 1979;86(5):854-63. https://doi.org/10.1016/S0161-642 0(79)37005-1.

30. Szucs-Farkas Z, Toth J, Balazs E, Galuska L, Burman KD, Karanyi Z, et al. Using morphologic parameters of extraocular muscles for diagnosis and follow-up of Graves' ophthalmopathy: diameters, areas, or volumes? AJR Am J Roentgenol. 2002;179(4):1005-10. https://doi.org/10.2214/ajr.179.4.1791005

31. Frueh BR, Musch DC, Garber FW. Lid retraction and levator aponeurosis defects in Graves' eye disease. Ophthalmic Surg. 1986;17(4):216-20.

32. Dickinson AJ, Perros P. Controversies in the clinical evaluation of active thyroid-associated orbitopathy: use of a detailed protocol with comparative photographs for objective assessment. Clin Endocrinol. 2001;55(3):283-303. https://doi.org/10.1046/j.1365-2265.2001.01349.x.

\section{Publisher's Note}

Springer Nature remains neutral with regard to jurisdictional claims in published maps and institutional affiliations.

Ready to submit your research? Choose BMC and benefit from:

- fast, convenient online submission

- thorough peer review by experienced researchers in your field

- rapid publication on acceptance

- support for research data, including large and complex data types

- gold Open Access which fosters wider collaboration and increased citations

- maximum visibility for your research: over $100 \mathrm{M}$ website views per year

At BMC, research is always in progress.

Learn more biomedcentral.com/submissions 